\title{
Analysis of Minor Component Segregation in Ternary Powder Mixtures
}

\author{
Maryam Asachi, Ali Hassanpour*, Mojtaba Ghadiri, and Andrew Bayly \\ School of Chemical and Process Engineering, University of Leeds, Leeds, LS2 9JT, UK
}

\begin{abstract}
In many powder handling operations, inhomogeneity in powder mixtures caused by segregation could have significant adverse impact on the quality as well as economics of the production. Segregation of a minor component of a highly active substance could have serious deleterious effects, an example is the segregation of enzyme granules in detergent powders. In this study, the effects of particle properties and bulk cohesion on the segregation tendency of minor component are analysed. The minor component is made sticky while not adversely affecting the flowability of samples. The segregation extent is evaluated using image processing of the photographic records taken from the front face of the heap after the pouring process. The optimum average sieve cut size of components for which segregation could be reduced is reported. It is also shown that the extent of segregation is significantly reduced by applying a thin layer of liquid to the surfaces of minor component, promoting an ordered mixture.
\end{abstract}

\section{Introduction}

In many industries dealing with dry powder handling operation, such as pharmaceutical, food and agriculture segregation is encountered, particularly during handling, transportation and storage of the product. Segregation could have significant impact on the quality as well as economics of the production. This phenomenon mainly happens due to difference in particles physical properties such as size, density and shape $[1,2]$. Some common examples of the segregation phenomenon that could cause problem are excessive concentration of the active ingredient in the mixture, such as insufficient bleach in a package of laundry detergent, taste variations in a powdered drink mixtures and variation of active pharmaceutical ingredients (API) content from tablet to tablet. Therefore, it is desirable to achieve a homogenous mixture [3].

One of the important factors affecting segregation phenomenon is variation in material properties. Particles with similar properties do not segregate, but segregation may take place as soon as there is variation in particles properties. Size distribution of particles is one of the main factors affecting this phenomenon. Usually a wider size distribution would result in more segregation [4]. Density variation of particles is another significant factor affecting segregation. Denser particles accumulate more in the centre of the powder mixtures like fine particles. Particle shape is also important for segregation. Spherical particles mostly behave like large and light particles and roll down the surfaces in a heap. In contrast, non-spherical particles almost collect closer to the centre of a heap similar to heavy particles [5].
Different segregation mechanisms could be seen as a result of particle interactions or influence of external forces causing the movement of particles in different directions as summarized by Bate [6].

Segregation of minor components such as expensive enzyme granules in detergent formulation could have significant economic impact on the production. By manipulating material properties, handling equipment, operational parameters and/or environmental conditions such as changing the bulk cohesion, it is possible to reduce segregation [4]. In this study, the segregation tendency of minor component (less than $3 \%$ weight ratio), such as enzyme placebo granules in a ternary mixture has been assessed. Desired properties for segregation reduction of enzyme placebo granules have been evaluated by finding the optimum material properties and/or controlling the particle cohesion. Effect of bulk cohesion on segregation reduction of enzyme placebo granules was studied by applying a thin layer of liquid to the surfaces of placebo granules. Different coating percentages of the coating material were assessed for the reduction of segregation. The optimum coating percentage is reported according the results of flowability of both bulk as well as enzyme placebo granules.

\section{Materials and Methodology}

As a model material system, a ternary washing powder mixture, comprising blue Tetra Acetyl Ethylene Diamine (TAED), white spray-dried synthetic detergent powder (Arial LS Diamond), known as Blown Powder (BP), and red enzyme placebo granules, is used. All the materials

Corresponding author: A.Hassanpour@leeds.ac.uk 
used in this investigation were obtained from Procter and Gamble (P\&G), Newcastle Innovation Centre, UK. To assess segregation, mixture of components $(40 \mathrm{~g})$ was poured in a box (dimension of $0.2 \times 0.2 \times 0.015 \mathrm{~m}$ ) to make a heap of washing powders. Segregation of components was evaluated using image processing of the picture captured from the front face of the box using Photoshop7 software. The experimental set-up used here mainly represents the granular piles building up in detergent industry, which are exposed to a relatively high extent of segregation. For the measurement of segregation index of component, the particle area was first determined, the pixels of the defined area was measured and converted to the fraction of components. Coefficient of variation (COV) concept was used as the segregation index in this study (Eq. 1).

$$
\text { Segregation index }=\mathrm{COV}=\frac{\sigma}{\bar{x}_{\text {Enzymeplacebo }}}
$$

where $\sigma$ is the standard deviation and $\bar{x}_{\text {Enzymeplacebo }}$ is the mean number fraction of the enzyme placebo granules in a defined set of grids within the whole area of the mixture. Shape characteristic of individual particles was determined using Scanning Electron Microscope (SEM) by Hitachi TM3030 Bench Top SEM. Coating of enzyme granules by polyethylene glycol (PEG, Pluriol E 600 ) was performed in a mixer. PEG was used as coating material due to its compatibility with detergent formulation. Flowability of the coated enzyme placebo granules as well as the bulk of powders was measured using Schulze shear cell device. Bulk tapped density measurement was also done by pouring the mixture of powders in a graduated cylinder and tapping the poured powders until the pack reached equilibrium. The mass and volume of the powder were then recorded to estimate the bulk tapped density.

\section{Results and Discussion}

\subsection{Optimum average sieve cut size for a well- mixed ternary mixture of laundry detergent powders}

The bulk tapped density of the mode size of enzyme placebo granules is measured as $1450 \mathrm{~kg} . \mathrm{m}^{-3}$ which is higher than $530 \mathrm{~kg} . \mathrm{m}^{-3}$ and $400 \mathrm{~kg} \cdot \mathrm{m}^{-3}$ measured for TAED and BP, respectively (sieve cut sizes between 300-600 $\mu \mathrm{m}$ ) suggesting enzyme granules are denser than BP and TAED particles.

To find a well-mixed ternary mixture, first $50 / 50$ weight ratio binary mixtures with the mode size of distribution of enzyme placebo granules (655 $\mu \mathrm{m}$, obtained by British standard sieve analysis presented in Fig. 1) and different average sieve cut sizes of $\mathrm{BP}$ and TAED (sieve cut sizes between 300-500 $\mu \mathrm{m}$ ) were separately prepared. The mixtures were then poured through a funnel fixed to the top side of the heap box to make heaps of binary mixture.

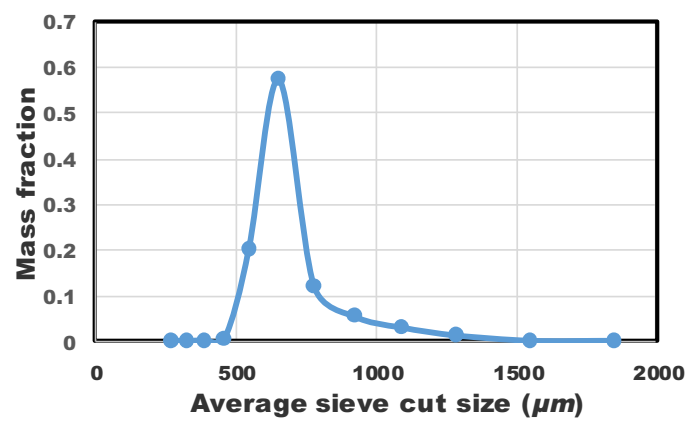

Fig. 1. Size distribution of enzyme placebo granules.

Figs. 2-b and 2-e demonstrate a relatively mixed condition of BP-placebo and TAED-placebo binary mixtures $(390 \mu m-655 \mu m)$. However, the binary mixtures of BP-placebo and TAED-placebo (328 $\mu \mathrm{m}-$ $655 \mu \mathrm{m})$ as shown in Figs. 2-a and 2-d, respectively undergone more segregation as compared to the previous cases. Size segregation of BP and TAED particles (328 $\mu m$ ) could dominate the density segregation of enzyme placebo granules (due to push-away effect) as shown in Figs. 2-a and 2-d. On the other hand, density segregation of enzyme placebo granules may dominate the size segregation of BP and TAED particles as shown in Figs. $2-\mathrm{c}$ and $2-\mathrm{f}$.
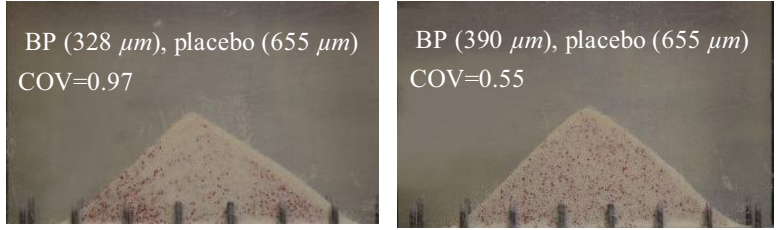

a)

b)

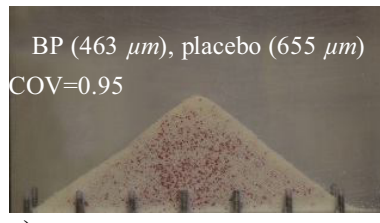

c)

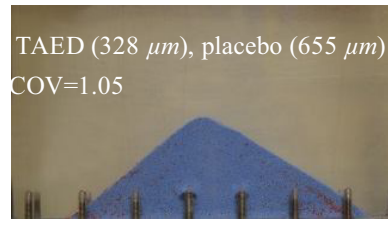

d)

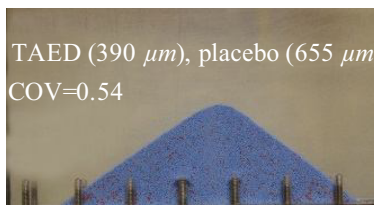

e)

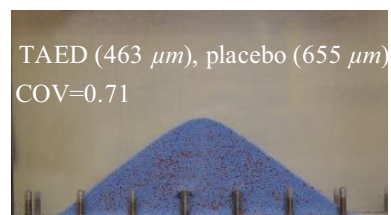

f)

Fig. 2. Segregation of binary mixtures of, a-c) BP-placebo, and d-f) TAED-placebo, having different average sieve cut sizes.

Two different ternary mixtures have been prepared with full size distribution of components (Fig. 3-a) and with the optimum sizes suggested earlier (Fig. 3-b) both with 92.6/5.55/1.85 weight ratio of BP, TAED and enzyme placebo granules. The segregation index of 
enzyme placebo granules, obtained by the image analysis as described earlier, decreased from 1.37 (full size distribution) to 0.76 by applying the optimum average sieve sizes for a mixed ternary mixture.

a)

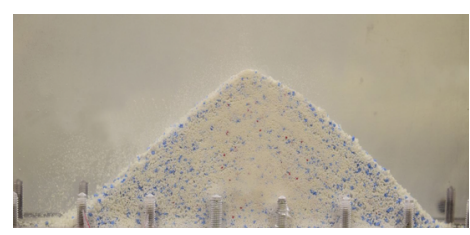

b)

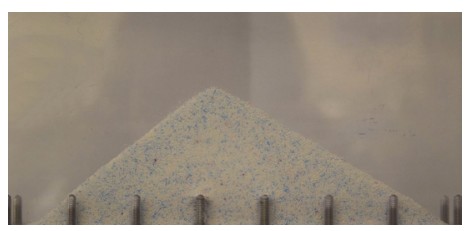

Fig. 3. a) Ternary mixtures containing full size distribution of BP, TAED and enzyme placebo granules with 92.6/5.55/1.85 weight ratio, b) mixed ternary mixture with the optimum sizes.

SEM pictures of components with the optimum average sieve cut sizes of particles described earlier is presented in Fig. 4. It can be seen that the enzyme placebo granules are more spherical than BP and TAED particles. It has been reported that spherical particles act like light, big and free flowing particles in mixtures of spherical and irregular particles and they tend to roll down the surface of the heap [5]. In contrast, irregular particles behave like heavy and fine particles building up into the centre of the heap [5]. However in this case, shape segregation of enzyme placebo granules could be balanced with other material properties of components, notably size and density.

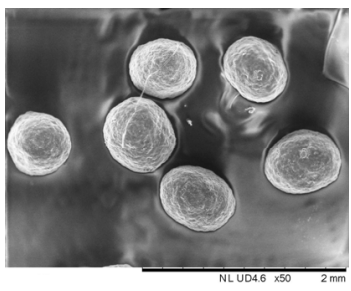

a)

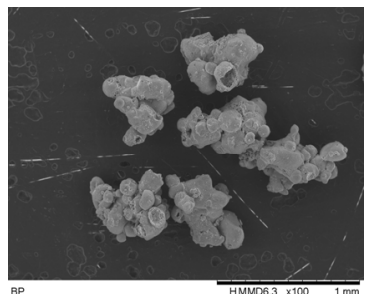

b)

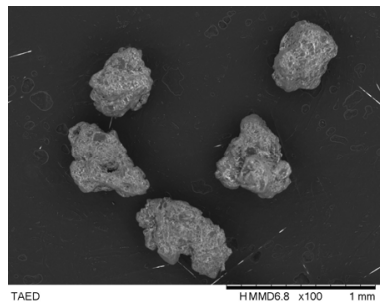

c)

Fig. 4. SEM pictures of the optimum average sieve cut size for a relatively mixed ternary mixture condition, a) enzyme placebo granules $(655 \mu m)$, b) BP $(390 \mu m), c)$ TAED (390 $\mu m)$.

With the results shown earlier, it could be concluded that a relatively mixed ternary mixture could be generated by the addition of denser enzyme placebo particles with its mode size $(655 \mu \mathrm{m})$ into the mixture of BP and TAED with average sieve cut size of $390 \mu \mathrm{m}$, balancing the effect of different material properties of particles. However, it may not be always practical to achieve the optimum average sieve cut sizes for a mixed ternary mixture to minimise segregation, particularly for industrial applications. Hence in this work, the effect of particle coating on the segregation reduction of enzyme placebo granules is investigated and reported in the next section.

\subsection{Effect of coating on the reduction of segregation of enzyme placebo granules}

To study the effect of coating on the reduction of segregation of enzyme placebo granules, different coating percentages of PEG $(0.5$ to $3.5 \%$ with the increment of $1 \%$ ) have been applied to placebo granules as described earlier. The coated enzyme placebo granules were added to the mixture of full size distribution BP and TAED particles to form a heap of ternary mixture with $92.6 / 5.55 / 1.85$ weight ratio of BP, TAED and enzyme placebo granules. The effect of coating on the segregation reduction of enzyme placebo granules is shown in Fig. 5-a. It could be observed that segregation of enzyme placebo granules decreased as coating level increased. However, the optimum coating level for a reasonable flowability of the particles should also be investigated. The flowability of both bulk mixture and coated enzyme placebo granules was analysed by the Schulze shear cell for which the results are shown in Fig. 5-b. It can be observed that the bulk flowability does not change significantly with the coating levels of 1.5 to $3.5 \%$ (remains in easy-flowing regime). However, the behaviour of enzyme placebo granules shifts to the cohesive regime, particularly at lower pre-shear with $3.5 \%$ coating level. According to the Figs. 5-a and 5-b, 2.5\% coating level could be enough to reduce segregation without compromising the flowability of both bulk and enzyme placebo granules. Ternary mixture with full size distribution of components after $2.5 \%$ coating level for enzyme placebo granules is shown in Fig. 6-a. It can be seen that some enzyme placebo particles (red particles) have reached to the surface of the heap, decreasing the overall segregation extent of enzyme placebo granules.

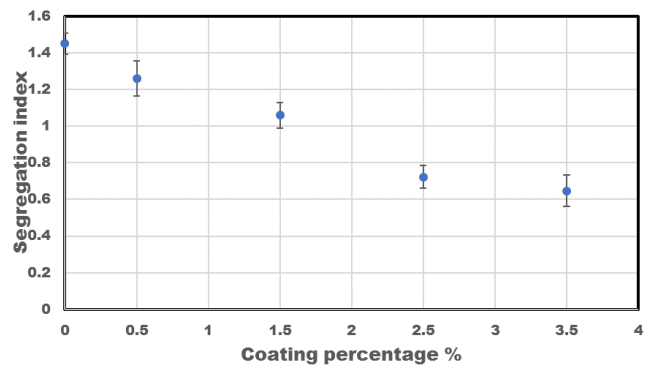

a) 


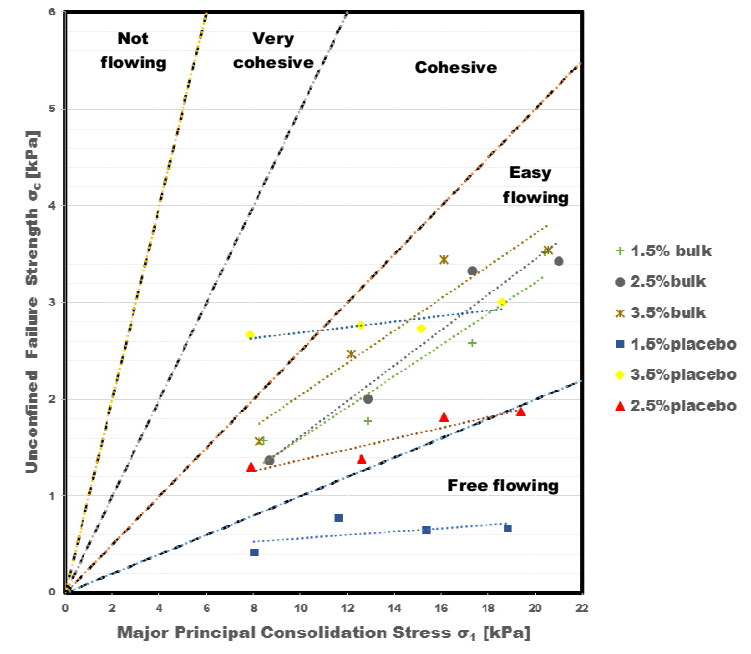

b)

Fig. 5. a) Segregation index versus coating percentage of PEG, b) flowability results of coated enzyme granules and bulk of detergent powders after coating.

The SEM picture of enzyme placebo granules after $2.5 \%$ coating percentage and after mixing with BP and TAED is illustrated in Fig. 6-b. It has been observed that BP particles adhere to the surface of coated enzyme placebo granules dispersing them within the bulk of mixture. Only fine BP particles adhere to the coated enzyme placebo granules and there is no evidence of TAED adherence. Adherence of fine BP particles onto the surfaces of the coated enzyme placebo granules modifies their shape and creates much rougher surfaces which further reduces their free movement in the mixture as compared to the case of free-flowing round enzyme placebo granules. This could lead to a reduction of the segregation of this component. Also, interlocking between particles could reduce the flowability of enzyme granules by the formation of liquid bridge between particles. It is recommended to test these hypotheses as part of future work. For instance, it would be useful to change the roughness of enzyme granules by other means to investigate its effect on the segregation.

a)

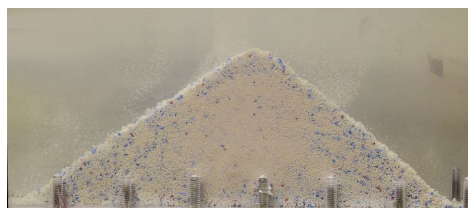

b)

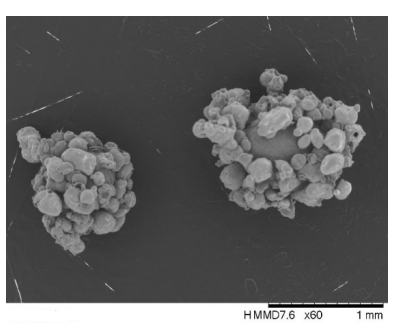

Fig. 6. a) Full size distribution ternary mixture after $2.5 \%$ coating of enzyme granules, b) SEM picture of coated enzyme placebo after mixing with BP and TAED.
In the literature, Discrete Element Method (DEM) simulation has been widely applied to study the kinematics of the flow in multi-component mixture of granular materials $[6,7]$. The dynamics of segregation of the ternary mixture studied in the current research can also be investigated using DEM technique, where effects of particle shape and surface roughness on the segregation tendency can be studied.

\section{Conclusions}

Segregation extent of minor component enzyme placebo granules has been investigated in this work. It is found that for enzyme placebo granules with the mode size of $655 \mu \mathrm{m}$, segregation can be reduced by using narrow sieve cuts of BP and TAED, with an average sieve cut size of $390 \mu \mathrm{m}$ in a ternary mixture of laundry detergent powders, as compared to the case of using their full size distribution. The effect of coating of enzyme placebo granules with a thin layer of sticky liquid on the segregation reduction of this component is also studied. From flowability test results, it is shown that $2.5 \%$ coating by PEG can adequately reduce the segregation of enzyme placebo granules without compromising the flowability of bulk and enzyme placebo granules itself.

\section{Acknowledgments}

We would like to acknowledge the financial support from AMSCI (The Advance Manufacturing Supply Chain Initiative is a government supply chain fund which is helping to rebuild British manufacturing prowess). In addition, the authors would like to thank Ms Claire Duckitt (Procter and Gamble, Newcastle Innovation Centre, UK) for project coordination.

\section{References}

1. J.K. Prescott, T.P. Garcia, Pharm. Technol. 25, 68 (2001).

2. X. He, X. Han, N. Ladyzhynsky, R. Deanne, Powder Technol. 236, 85 (2013).

3. A.N. Huang, H.P. Kuo, Adv. Powder Technol. 25, 163 (2014)

4. P. Tang, V.M. Puri, Part. Sci. Technol. 22, 321 (2004).

5. G.G. Enstad, Handbook of Conveying and Handling of Particulate Solids. 10, 589 (2001).

6. L. Bates, User guide to segregation. First edition, British Materials Handling Board, 1997.

7. B. Marks, P. Rognon, I. Einav, J. Fluid Mech. 690, 499 (2012).

8. J. M. N. T. Gray and C. Ancey, J. Fluid Mech. 678, 535 (2011). 